\title{
UN CAS NOUVEAU DE MYIASE DU TUBE DIGESTIF CAUSÉ PAR LA LARVE DE PSYCHODA B. PUNCTATA CURT.
}

\author{
Par Jô K. OKADA
}

Je voudrais exposer ici un cas nouveau de myiase ; il s'agit d'une larve de diptère que m'a envoyée M. le docteur Haruno, un de mes amis du Japon. D'après ce qu'il m'a écrit, cette larve lui avait été donnée lors d'un séjour dans son pays natal par un médecin de ses parents résidant en province. "Une jeune fille de 17 ans, souffrant de l'estomac, vint consulter le médecin. Quelques jours après, cette jeune fille, prise de vomissements, constata, dans ses matières, la présence de corps doués de mouvement. Elle lui apporta quẻlques exemplaires. D'ailleurs elle a été prise de vomissements répétés avant et après cette visite. Le médecin s'est rendu compte qu'il s'agissait là de parasites mais il n'a pu, au premier abord, les identifier. Il les a conservés dans l'alcool et les a confiés à mon ami pour qu'il en poursuive l'identification à Paris. "

Le nombre des parasites envoyés était d'une cinquantaine et la taille des plus grands était d'environ $3 \mathrm{~mm}$. Le corps, de couleur jaunàtre et de forme cylindrique s'amincissant aux deux extrémités, est constitué par 12 anneaux. Une extrémité, de couleur brunâtre, qui est la tête de l'animal, présente une induration de la chitine et porte une paire d'yeux. La lèvre supérieure est bien développée, la lèvre inférieure possède de petites dents en forme de scie ; l'extrémité de la mandibule est divisée en quatre parties. Le deuxième anneau présente de chaque côté un stigmate proéminent de couleur brun sombre. Du troisième au dixième anneau, il n'y a plus qu'une suite de segments cylindriques sans aucune caractéristique spéciale. Le onzième et le douzième anneaux, au contraire, sont plus allongés et moins larges que les autres et portent chacun trois plaques indurées, longitudinales, de couleur brun sombre. L'extrémité de la queue est aussi colorée et se dresse obliquement ; elle se termine par quatre protubérances.

Il paraît évident qu'il s'agit là de la larve d'un diptère : étant donné l'aspect extérieur et surtout les taches brunes des derniers anneaux, on a pu se rendre compte qu'on se trouvait en présence de la larve de Psychoda $b$. punctata Curt.

Au Japon, le Psychoda a pour habitat les terres humides et AnNales de Parasitologie, T. V, $\mathrm{N}^{\circ} 2 .-1^{\mathrm{er}}$ avril $1927, \mathrm{p}, 105-106$. 
salines. Il lui est donc possible de déposer ses œufs sur des aliments conservés dans ces parages. Cependant, cet exemple de myiase nous a paru exceptionnel. Les faits : que la malade a vomi les larves et que ces dernières avaient une taille inférieure à celle atteinte à maturité ( $7 \mathrm{~mm}$. environ), semblent prouver que ce parasitisme est temporaire. Mais on n'a pas d'autres observations de myiase causée par cette larve de Psychoda et il s'agissait d'une jeune fille anémiée. Il nous parait naturel de considérer ce parasitisme comme accidentel et même comme un eas particulier. L'œuf de Psychoda ou la larve, absorbés avec les aliments, succomberont dans l'estomac, si l'on se trouve en bonne santé ; mais si l'on a une mauvaise digestion et si cette larve devient susceptible de résistance contre les liquides de l'estomac, il pourra se produire, parfois, une myiase.

Si nous admettons cette hypothèse, l'étude des mœurs du Psychoda au Japon ne nous parait pas inutile. On doit surtout rappeler que certaines larves de diptère se sont une fois développées dans un tuyau de conduite d'eau de la ville d'Odaru (à Hokkaidô ou Ezo), dont l'installation, dit-on, n'était pas parfaite. M. le professeur Matsumura a élevé les larves qu'il avait trouvées dans ce tuyau et il a obtenu deux espèces de diptères : Chironomus aquæducti Mats. et Epolanylarsus japonibius Mats. Il a dit que les larves de Psychoda vivent aussi dans le réservoir de la conduite d'eau et que si le tuyautage n'est pas rigoureusement étanche, il peut arriver qu'elles y pénètrent (Dóbutsu Gaku Zasshi, 1917, vol. 29, p. 7).

Quoi qu'il en soit, il est très intéressant au point de vue de l'hygiène sociale comme au point de vue parasitologique, de savoir qu'une larve de Psychoda est capable de se développer dans les tuyaux de conduites d'eau et de pénétrer accidentellement dans le corps humain.

Dans le cas de la myiase que nous signalons, on ignore si les larves ont été absorbées ou si ses œufs, déposés sur les aliments, ont éclos dans l'estomac et s'y sont développés. " Cependant, étant donné ce que l'on sait de la malade, on peut dire qu'elle n'aurait pas pris d'aliments avariés portant de nombreuses larves encore grouillantes devant ses yeux -n (d'après M. Haruno). Il est plus probable d'admettre qu'elle a absorbé des œufs déposés sur les aliments.

Je tiens ici à exprimer mes sincères remerciements à $M$. le docteur Haruno qui m'a communiqué les larves et à M. le professeur Komai, de l'Université de Kyôto, qui a bien voulu m'envoyer l'ouvrage du professeur Matsumura cité plus haut. 